\title{
Prognostic effect of body mass index in patients with advanced NSCLC treated with chemoimmunotherapy combinations
}

\author{
Alessio Cortellini (D) , ${ }^{1}$ Biagio Ricciuti (D) , ${ }^{2}$ Victor R Vaz, ${ }^{2}$ Davide Soldato, ${ }^{3}$ \\ Joao V Alessi (1) , ${ }^{2}$ Filippo G Dall'Olio, ${ }^{3}$ Giuseppe L Banna, ${ }^{4}$ \\ Sethupathi Muthuramalingam, ${ }^{5}$ Samuel Chan, ${ }^{6}$ Margarita Majem (1) , ${ }^{7}$ Aida Piedra, ${ }^{7}$ \\ Giuseppe Lamberti (i) , ${ }^{8}$ Elisa Andrini, ${ }^{8}$ Alfredo Addeo, ${ }^{9}$ Alex Friedlaender, ${ }^{9}$ \\ Francesco Facchinetti, ${ }^{10}$ Teresa Gorría, ${ }^{11}$ Laura Mezquita (D) , ${ }^{12}$ Delphine Hoton, ${ }^{13}$ \\ Lacroix Valerie, ${ }^{14}$ Frank Aboubakar Nana, ${ }^{15}$ James Artingstall, ${ }^{16}$ Charles Comins, ${ }^{16}$ \\ Massimo Di Maio, ${ }^{17}$ Andrea Caglio, ${ }^{17}$ Judith Cave, ${ }^{18}$ Hayley McKenzie, ${ }^{18}$ \\ Thomas Newsom-Davis, ${ }^{19}$ Joanne S Evans, ${ }^{1}$ Marcello Tiseo, ${ }^{20,21}$ \\ Antonio D'Alessio, ${ }^{1,22}$ Claudia A M Fulgenzi, ${ }^{1,23}$ Benjamin Besse, ${ }^{3,24}$ \\ Mark M Awad (D) ,2 David J Pinato (D) ${ }^{1,25}$
}

To cite: Cortellini A, Ricciuti B, Vaz VR, et al. Prognostic effect of body mass index in patients with advanced NSCLC treated with chemoimmunotherapy combinations. Journal for ImmunoTherapy of Cancer 2022;10:e004374. doi:10.1136/ jitc-2021-004374

- Additional supplemental material is published online only. To view, please visit the journal online (http://dx.doi.org/10. 1136/jitc-2021-004374).

Accepted 11 January 2022

Check for updates

(C) Author(s) (or their employer(s)) 2022. Re-use permitted under CC BY-NC. No commercial re-use. See rights and permissions. Published by BMJ.

For numbered affiliations see end of article.

Correspondence to Dr Alessio Cortellini; alessiocortellini@gmail.com

\section{ABSTRACT}

Introduction It has been recognized that increasing body mass index (BMI) is associated with improved outcome from immune checkpoint inhibitors (ICls) in patients with various malignancies including non-small cell lung cancer (NSCLC). However, it is unclear whether baseline BMI may influence outcomes from first-line chemoimmunotherapy combinations. Methods In this international multicenter study, we evaluated the association between baseline BMI, progression-free survival (PFS) and overall survival (OS) in a cohort of patients with stage IV NSCLC consecutively treated with first-line chemoimmunotherapy combinations. BMI was categorized according to WHO criteria.

Results Among the 853 included patients, 5.3\% were underweight; $46.4 \%$ were of normal weight; $33.8 \%$ were overweight; and $14.5 \%$ were obese. Overweight and obese patients were more likely aged $\geq 70$ years $(p=0.00085)$, never smokers $(p<0.0001)$, with better baseline Eastern Cooperative Oncology Group - Performance Status ( $p=0.0127)$, and had lower prevalence of central nervous system $(p=0.0002)$ and liver metastases $(p=0.0395)$. Univariable analyses showed a significant difference in the median OS across underweight (15.5 months), normal weight (14.6 months), overweight (20.9 months), and obese (16.8 months) patients (log-rank: $p=0.045$, log rank test for trend: $p=0.131$ ), while no difference was found with respect to the median PFS (log-rank for trend: $\mathrm{p}=0.510$ ). Neither OS nor PFS was significantly associated with baseline BMI on multivariable analysis.

Conclusions In contrast to what was observed in the context of chemotherapy-free ICl-based regimens, baseline BMI does not affect clinical outcomes from chemoimmunotherapy combinations in patients with advanced NSCLC.

\section{INTRODUCTION}

Increasing evidence suggests the presence of an obesity-driven proinflammatory state in patients with cancer, with positive implications with regard to clinical benefit from immune checkpoint inhibitors (ICIs). ${ }^{1-3}$ In patients with non-small cell lung cancer (NSCLC), baseline obesity is associated with an incremental survival benefit with programmed death-1 (PD-1)/ programmed death-ligand 1 (PD-L1) inhibitors compared with normal-weight patients, a finding confirmed across different treatment lines and levels of PD-L1 tumor expression. ${ }^{45}$ In a prior study evaluating patients with advanced NSCLC treated with either first-line pembrolizumab monotherapy or standard chemotherapy, we showed that the positive effect of body mass index (BMI) on oncological outcomes was restricted to immunotherapy recipients, lending further credence to the view that obesity may exert an immune modulatory rather than a simply prognostic role. ${ }^{6}$

Considerable research efforts are under way to identify tumorous and host determinants of response and survival in the context of chemoimmunotherapy combinations, which have significantly improved the first-line treatment landscape of $\mathrm{NSCLC}^{78}$; however, to date, there is no clear evidence about the role of baseline BMI in this setting.

\section{METHODS}

In this international multicenter study, we evaluated the association between baseline BMI and clinical outcomes in a cohort 
Table 1 Patients' characteristics at baseline for the overall cohort and according to body mass index WHO categories

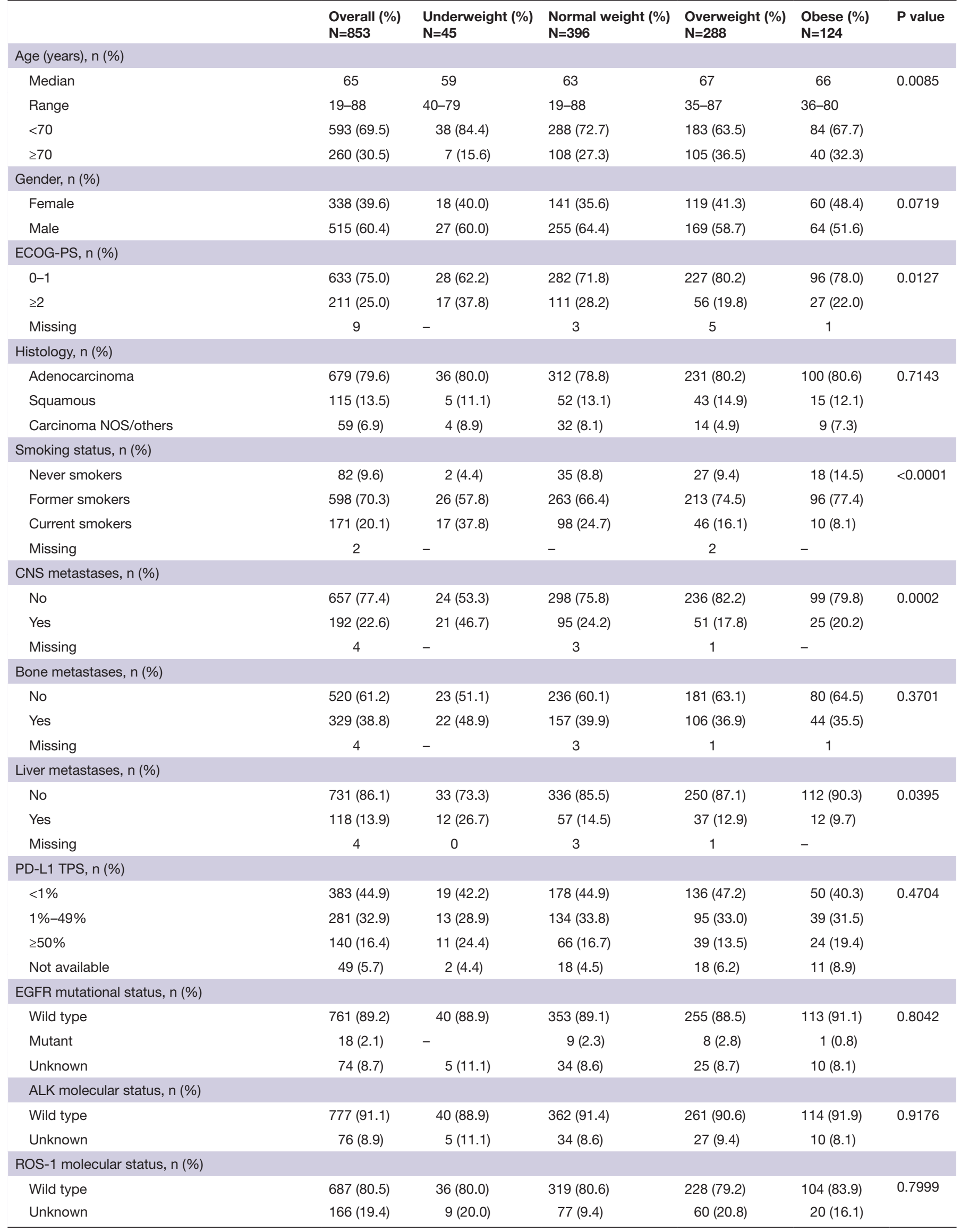


Table 1 Continued

\begin{tabular}{|c|c|c|c|c|c|c|}
\hline \multicolumn{7}{|l|}{ KRAS molecular status, $\mathrm{n}(\%)$} \\
\hline Wild type & $338(39.6)$ & $14(31.1)$ & $173(43.7)$ & $114(39.6)$ & $37(29.8)$ & 0.0011 \\
\hline Mutant & $226(26.5)$ & $14(31.1)$ & $85(21.5)$ & $75(26.0)$ & $52(41.9)$ & \\
\hline \multicolumn{7}{|l|}{ STK11 molecular status, $\mathrm{n}(\%)$} \\
\hline Wild type & $247(29.0)$ & $9(20.0)$ & $115(29.0)$ & $86(29.9)$ & $37(29.8)$ & 0.7273 \\
\hline Mutant & $91(10.7)$ & $5(11.1)$ & $39(9.8)$ & $30(10.4)$ & $17(13.7)$ & \\
\hline \multicolumn{7}{|l|}{ KEAP-1 molecular status, $n(\%)$} \\
\hline Wild type & $244(28.6)$ & $12(26.7)$ & $105(26.5)$ & $84(29.2)$ & $43(34.7)$ & 0.4988 \\
\hline Mutant & $67(7.9)$ & $2(4.4)$ & $36(9.1)$ & $19(6.6)$ & $10(8.1)$ & \\
\hline Unknown & $542(63.5)$ & $31(68.9)$ & $255(64.4)$ & $185(64.2)$ & $71(57.3)$ & \\
\hline \multicolumn{7}{|l|}{ TP53 molecular status, n (\%) } \\
\hline \multicolumn{7}{|l|}{ Median TMB (mut/megabase) } \\
\hline Median (range) & $\begin{array}{l}9.1(1.0- \\
67.6)\end{array}$ & $12.2(5.3-36.5)$ & $9.1(1.2-67.6)$ & $8.4(1.0-25.1)$ & $8.4(1.3-25.1)$ & 0.1590 \\
\hline$<10$ & $148(59.7)$ & $3(27.3)$ & $68(60.2)$ & $49(61.2)$ & $28(63.6)$ & \\
\hline$\geq 10$ & $100(40.3)$ & $8(72.7)$ & $45(39.8)$ & $31(38.7)$ & $16(36.4)$ & \\
\hline Available patients & 248 & 11 & 113 & 80 & 44 & \\
\hline \multicolumn{7}{|l|}{ Other potentially targetable oncogenes* } \\
\hline Mutant & $61(7.1)$ & $1(2.2)$ & $31(7.8)$ & $23(7.9)$ & $6(4.8)$ & - \\
\hline \multicolumn{7}{|l|}{ Regimen } \\
\hline
\end{tabular}

*Includes HER2 (available for 466 patients), MET (available for 477 patients), BRAF (available for 526 patients) and RET (available for 448 patients). ALK, anaplastic lymphoma kinase; CNS, central nervous system; ECOG-PS, Eastern Cooperative Oncology Group-Performance Status; EGFR, epidermal growth factor receptor; PD-L1, programmed death-ligand 1; TMB, tumor mutational burden; TPS, Tumor Proportion Score.
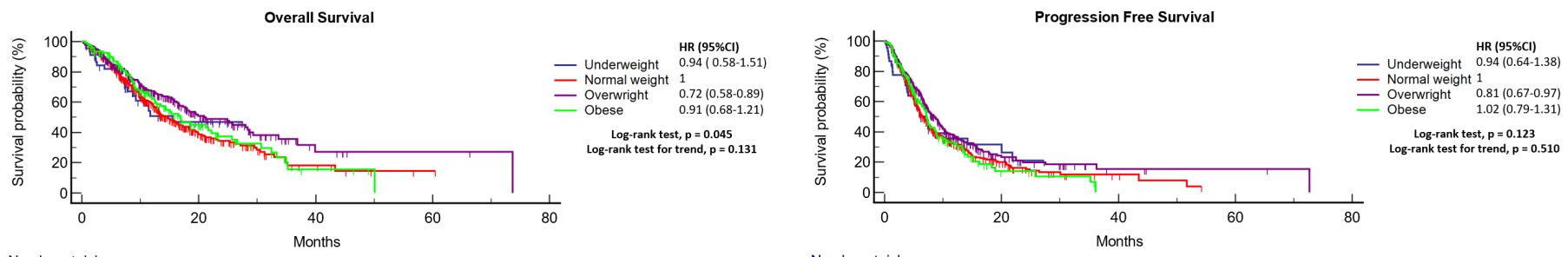

Number at risk

Underweught

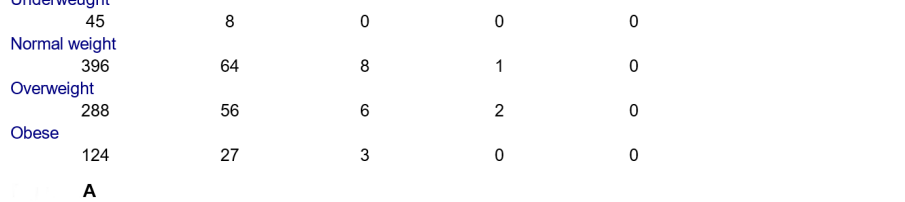

\begin{tabular}{rr} 
Number at ris \\
Underweight \\
45 \\
Normal weigh \\
396 \\
\multicolumn{2}{c}{396} \\
Overweight \\
\multicolumn{1}{c}{288} \\
Obese & 124 \\
&
\end{tabular}

Figure 1 Kaplan-Meier survival estimates. (A) Overall survival: underweight: 15.5 months (95\% Cl 8.8 to $15.5,20$ events), normal weight: 14.6 months $(95 \% \mathrm{Cl} 13.1$ to $17.2,207$ events), overweight: 20.9 months (95\% Cl 17.3 to $28.7,116$ events), obese: 16.8 months $(95 \% \mathrm{Cl} 12.5$ to $23.2,64$ events). (B) Progression-free survival: underweight: 6.9 months $(95 \% \mathrm{Cl} 4.0$ to 14.2, 30 events), normal weight: 6.6 months (95\% Cl 5.8 to 7.3, 283 events), overweight: 8.4 months (95\% Cl 7.2 to $9.7,182$ events), obese: 7.2 months (95\% $\mathrm{Cl} 6.0$ to $8.6,87$ events). 
Table 2 Fixed multivariable analysis for risk of disease progression (PFS) and death (OS)

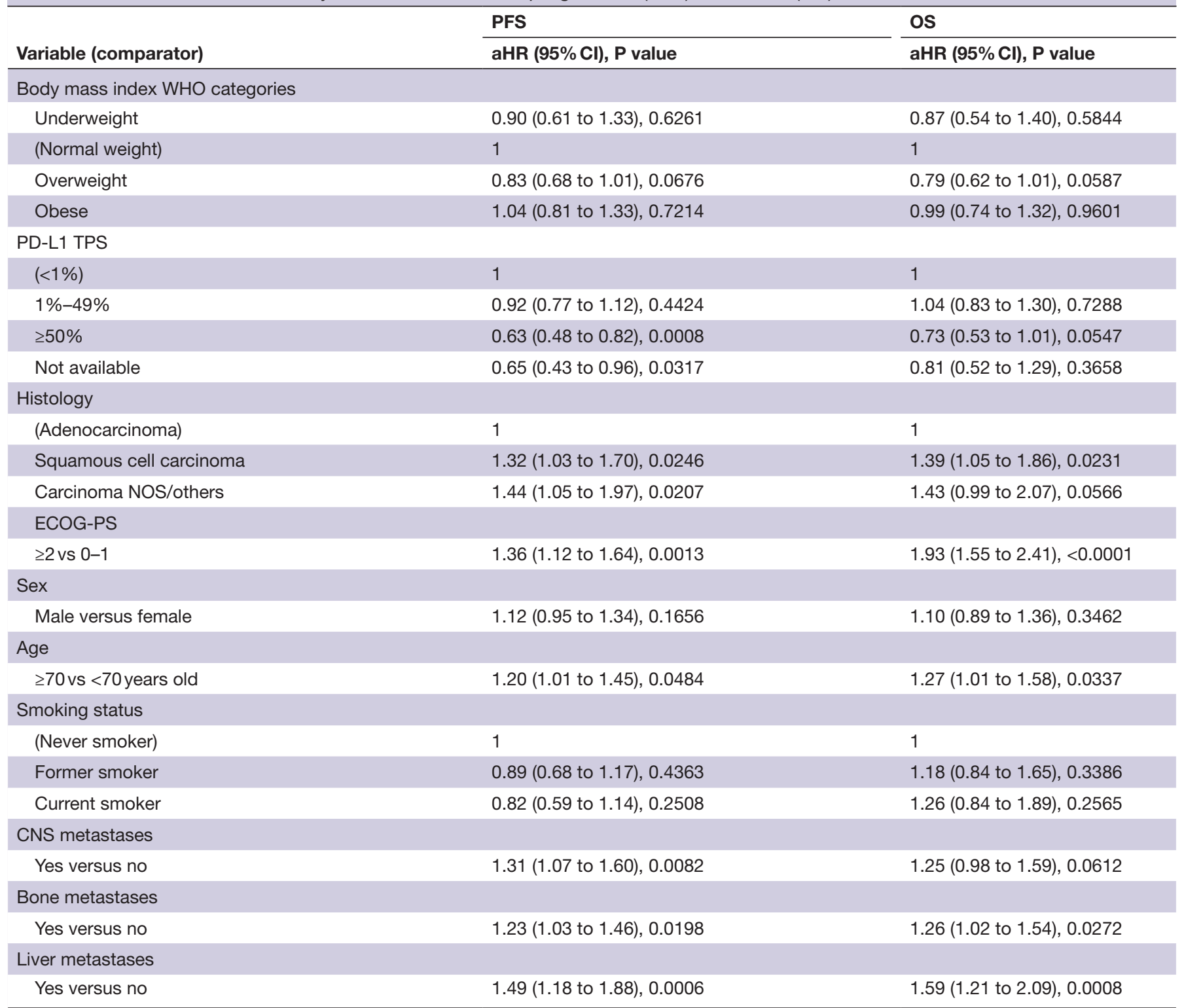

838 patients included due to missing values.

aHR, adjusted HR; CNS, central nervous system; ECOG-PS, Eastern Cooperative Oncology Group-Performance Status; OS, overall survival; PDL1, programmed death-ligand 1; PFS, progression- free survival.

of patients with stage IV NSCLC treated with first-line chemoimmunotherapy combinations.

In total, 15 institutions across seven countries participated in the study (online supplemental table 1) and retrospectively included patients treated from December 2014 to August 2021, with data cut-off in November 2021.

Patients with oncogene-addicted disease previously treated with targeted agents only were considered eligible. Clinical endpoints included overall survival (OS) and progression-free survival (PFS). Tumor imaging was assessed at baseline and during treatment at participating institutions, with a frequency of 8-12 weeks according to local practice. Investigators from participating centers independently reviewed disease response following Response Evaluation Criteria in Solid Tumors (RECIST) criteria V.1.1. PFS and OS were measured from treatment initiation to disease progression and/or death. Patients without documented disease progression were censored on the date of last imaging follow-up.

\section{Evaluation of baseline BMI}

Patients' BMI was calculated using the formula of weight/height ${ }^{2}$ (kilogram/square meter) and categorized according to the WHO categories: underweight (BMI $<18.5)$, normal weight $(18.5 \leq \mathrm{BMI} \leq 24.9)$, overweight $(25 \leq \mathrm{BMI} \leq 29.9)$, and obese (BMI $\geq 30)$. Weight and height were retrieved from patient medical records at baseline and derived within 30 days of treatment initiation.

First, we evaluated the distribution of patients' characteristics across BMI subgroups, in order to explore 
possible associations between baseline BMI and clinicopathological features. Subsequently, we assessed the impact of baseline BMI on outcome using univariable analysis. Considering the results of the univariable analysis, we then used fixed multivariable regression models to further validate our findings. Covariates were chosen on a clinical prioritization basis, in view of their known prognostic role, including PD-L1 tumor expression ( $\geq 50 \%$ vs $1 \%-49 \%$ vs negative vs not available), primary tumor histology (adenocarcinoma vs squamous cell carcinoma vs carcinoma not otherwise specified-/others), Eastern Cooperative Oncology Group-Performance Status (ECOG-PS, 0-1 vs $\geq 2$ ), sex (male vs female), age ( $<70$ vs $\geq 70$ years old), smoking status (current smokers vs former smokers vs never smokers), presence of central nervous system (CNS) metastases (yes vs no), bone metastases (yes vs no), and liver metastases (yes vs no).

Considering the incremental benefit reported with ICIs for obese patients over normal-weight patients in PD-L1 selected populations, ${ }^{56}$ we added two exploratory analyses including patients with PD-L1 negative and positive tumors, and with PD-L1 high ( $\geq 50 \%)$ and low (1\%-49\%) tumor expression, respectively. An additional ancillary analysis including only patients with an ECOG-PS of $0-1$ was also performed. In all the regression analyses, normal-weight patients were considered as the comparator group.

\section{Statistical analysis}

Baseline patients' characteristics were reported with descriptive statistics as appropriate. The $\chi^{2}$ and test was used to compare categorical variables. PFS/OS were evaluated and compared using the Kaplan-Meier method, the log-rank test, and the log-rank test for trend. Duration of follow-up was calculated according to the reverse KaplanMeier method. Cox proportional hazards regression was used for the multivariable analysis of PFS and OS and to compute the HRs with 95\% CIs. Missing values for clinicopathological characteristics included in the regression analyses were excluded from the descriptive analysis and the multivariable models. All $\mathrm{p}$ values were two-sided and CIs set at the $95 \%$ level, with significance predefined to be at $<0.05$. All statistical analyses were performed using the MedCalc Statistical Software V.20 (MedCalc Software, Ostend, Belgium; https://www.medcalc.org; 2021).

\section{RESULTS}

After the exclusion of 26 patients due to missing BMI data, 853 patients were included in the present analysis. Characteristics of the study population stratified by WHO BMI subgrouping are summarized in table 1 .

In total, 45 patients $(5.3 \%)$ were underweight; 396 (46.4\%) were normal weight; 288 (33.8\%) were overweight; and $124(14.5 \%)$ were obese. A total of 211 patients had a baseline ECOG-PS of $\geq 2$ (25.0\%). PD-L1 tumor expression was evaluable in 804 patients (94.2\%), showing a Tumor Proportion Score of $\geq 50 \%$ in 140 (16.4\%), $1 \%-49 \%$ in
$281(32.9 \%)$, and $<1 \%$ in $383(44.9 \%)$ patients, respectively. Most of the patients were epidermal growth factor receptor $(762,89.2 \%)$, anaplastic lymphoma kinase (777, $91.1 \%)$, and ROS proto-oncogene $1(687,80.5 \%)$ wild type. Other molecular findings relevant for ICI outcomes were also reported (when available).

Several baseline clinicopathological features were significantly different across BMI categories. Overweight and obese patients were more likely aged $\geq 70$ years $(p=0.00085)$ and never smokers $(p<0.0001)$, with better baseline ECOG-PS $(\mathrm{p}=0.0127)$ and lower prevalence of liver metastases $(p=0.0395)$. Prevalence of baseline CNS metastases was also different across BMI categories $(\mathrm{p}=0.0002)$, with the lowest prevalence reported for the overweight subgroup (17.8\%), as well as the distribution of the Kirsten rat sarcoma virus (KRAS) mutational status $(\mathrm{p}=0.0011)$, with the highest prevalence of mutant patients within the obese subgroup (41.9\%).

With a median follow-up of 17.5 months (95\% CI 15.9 to 18.7), the median PFS and OS of the entire cohort were 7.2 months (95\% CI 6.7 to $7.8,582$ events) and 16.8 months (95\% CI 15.2 to $19.3,407$ events), respectively.

The median OS across underweight, normal weight, overweight, and obese patients were 15.5 months (95\% CI 8.8 to 15.5, 20 events), 14.6 months (95\% CI 13.1 to $17.2,207$ events), 20.9 months (95\% CI 17.3 to 28.7, 116 events), and 16.8 months (95\% CI 12.5 to 23.2, 64 events), respectively (log rank: $\mathrm{p}=0.045, \log$-rank test for trend: $\mathrm{p}=0.131$; figure $1 \mathrm{~A}$ ), while the median PFS across underweight, normal weight, overweight and obese patients were 6.9 months (95\% CI 4.0 to 14.2, 30 events), 6.6 months (95\% CI 5.8 to 7.3 , 283 events), 8.4 months (95\% CI 7.2 to $9.7,182$ events), and 7.2 months (95\% CI 6.0 to $8.6,87$ events), respectively (log rank: $\mathrm{p}=0.123, \log$ rank test for trend: $\mathrm{p}=0.510$; figure $1 \mathrm{~B}$ ).

Table 2 reports the multivariable analyses for PFS and OS. No association was confirmed between baseline BMI and clinical outcomes. PD-L1 tumor expression, ECOGPS, primary tumor histology, age, CNS, and bone and liver metastases were confirmed significant determinants of PFS, while ECOG-PS, primary tumor histology, age, bone and liver metastases were confirmed significant determinants for OS.

Online supplemental figure 1 and online supplemental figure 2 summarize the exploratory analyses including patients with PD-L1 negative and positive tumors, and with PD-L1 of $\geq 50 \%$ and $1 \%-49 \%$ tumor expression, according to which baseline BMI was not associated with clinical outcomes in any of the PD-L1 expression subgroups.

The ancillary analysis including only patients with a good PS (ECOG-PS 0-1) is summarized in online supplemental figure 3; no association between baseline BMI and OS/PFS was confirmed.

\section{DISCUSSION}

In this study, we did not find any significant association between baseline BMI and clinical outcomes in patients 
with NSCLC treated with first-line chemoimmunotherapy combinations, regardless of PD-L1 tumor expression.

The addition of chemotherapy to ICI is known to enhance tumor antigenicity and can improve treatment efficacy. This changing algorithm has led to the shifting of some of the associative paradigms we observed with chemotherapy-free, ICI-based regimens. For instance, our group recently showed that a previous antibiotic therapy does not impair treatment outcomes in patients with NSCLC treated with chemoimmunotherapy combinations, as reported with single-agent ICI instead. ${ }^{910}$ The absence of a BMI-dependent effect on clinical outcome mirrors these findings and highlights how the host determinants of benefit from ICI might have different roles depending on the specific treatment modality. In the context of single-agent ICI regimens, obesity has been interpreted as a driver of reduced responsivity of peripheral T cells, due to the a dysfunctional PD-1/PD-L1-driven immune exhaustion, which could explain the magnified effect of PD-1/PD-L1 inhibitors in restoring T-cell activity in obese individuals. ${ }^{3}$ The addition of the chemotherapy backbone could potentially mitigate this mechanism through the enhanced immunogenicity, which minimizes in turn the role of BMI and obesity.

Improved outcome has been documented for eversmokers in the context of single-agent ICI. ${ }^{11}$ Interestingly, in our population, overweight and obese patients were more likely never smokers. This could be partially linked to the alleged historical association between the smoking behavior and body weight/fat distribution. ${ }^{12}{ }^{13}$ However, in our population and in chemoimmunotherapy trials as well, the role of the smoking status as a strong driver of improved outcomes with chemotherapy-free ICI regimens has also been dimensioned. ${ }^{14}$

Evidence for a positive prognostic role for a high baseline BMI was already described in patients with NSCLC treated with first-line chemotherapy during the "preICI era'. ${ }^{15}$ Several evidence highlights that a systemic inflammatory overactivation plays a central role as cancer cachexia mechanism, ${ }^{16}$ and in an aggressive disease such as metastatic lung cancer, baseline nutrition, weight loss, and performance status were historically considered closely intertwined. ${ }^{17}$ From this perspective, the 30-day time window for baseline BMI data collection could even be considered as a partial limitation to our study.

In previous reports including single-agent ICI recipients, a linear trend between increasing BMI and incremental benefit was reported, with obese patients experiencing the best outcome ${ }^{5}$; in this cohort, overweight patients are those who achieved the longest survival in absolute terms. Importantly, we also found an association between increasing BMI and better ECOG-PS/lower burden of disease, which are major drivers of better outcome with ICIs, ${ }^{18}$ with the lowest prevalence of patients with poor performance status for the overweight group.

Despite acknowledging several limitations, mainly coming from the retrospective design, the lack of matched control cohorts receiving first-line single-agent immunotherapy and chemotherapy, the lack of centralized data/imaging review, and incomplete molecular profile for all the patients, our study provides a powered analysis and reliable evidence about the absence of a significant role for the baseline BMI in this setting. As additional limitation, the lack of comorbidity data, especially those closely linked to dysmetabolism, such as cardiopulmonary diseases, hypertension, diabetes mellitus, and dyslipidemia, also needs to be mentioned.

Our findings suggest that, in contrast to what has been reported in the context of single-agent ICI, baseline BMI should not be taken into consideration when counseling patients with NSCLCfor a first-line chemoimmunotherapy.

\section{Author affiliations}

${ }^{1}$ Department of Surgery and Cancer, Imperial College London, London, UK ${ }^{2}$ Lowe Center for Thoracic Oncology, Dana Farber Cancer Institute, Boston, Massachusetts, USA

${ }^{3}$ Cancer Medicine Department, Gustave Roussy, Villejuif, France

${ }^{4}$ Candiolo Cancer Institute, FPO-IRCCS, Candiolo, Italy

${ }^{5}$ Oncology Department, United Lincolnshire Hospital NHS Trust, Lincoln, UK ${ }^{6}$ Oncology Department, Queen Alexandra University Hospital, Portsmouth Hospitals NHS Trust, Portsmouth, UK

${ }^{7}$ Medical Oncology Department, Hospital de la Santa Creu i Sant Pau, Barcelona, Spain

${ }^{8}$ Department of Experimental, Diagnostic and Specialty Medicine, S. Orsola-Malpighi University Hospital, Alma Mater Studiorum University of Bologna, Bologna, Italy ${ }^{9}$ Medical Oncology, University Hospital of Geneva, Geneva, Switzerland ${ }^{10}$ Biomarqueurs Prédictifs et Nouvelles Stratégies Thérapeutiques en Oncologie, Université Paris-Saclay, Institut Gustave Roussy, Inserm, Villejuif, France

${ }^{11}$ Department of Medical Oncology, Hospital Clinic, Barcelona, Spain

${ }^{12}$ Medical Oncology Department, Hospital Clinic de Barcelona, Barcelona, Spain

${ }^{13}$ Department of Pathology, Cliniques universitaires Saint-Luc, Bruxelles, Belgium

${ }^{14}$ Department of Cardiovascular and Thoracic Surgery, IREC, Cliniques Universitaires Saint-Luc, Bruxelles, Belgium

${ }^{15}$ Institut de Recherche Expérimentale et Clinique (IREC), Pôle de Pneumologie, ORL et Dermatologie (PNEU), Université catholique de Louvain (UCLouvain), Brussels, Belgium

${ }^{16}$ Bristol Haematology and Oncology Centre, Bristol, UK

${ }^{17}$ Department of Oncology, University of Turin and Mauriziano Hospital, Turin, Italy

${ }^{18}$ Department of Medical Oncology, University Hospital Southampton NHS

Foundation Trust, Southampton, UK

${ }^{19}$ Department of Oncology and National Centre for HIV Malignancies, Chelsea and Westminster Hospital, London, UK

${ }^{20}$ Department of Medicine and Surgery, University of Parma, Parma, Italy

${ }^{21}$ Medical Oncology Unit, University Hospital of Parma, Parma, Italy

${ }^{22}$ Department of Biomedical Sciences, Humanitas University, Pieve Emanuele (Milan), Italy

${ }^{23}$ Department of Medical Oncology, University Campus Bio-Medico, Rome, Italy

${ }^{24}$ School of Medicine, University Paris-Saclay, Villejuif, France

${ }^{25}$ Division of Oncology, Department of Translational Medicine, University of Piemonte Orientale, Novara, Italy

Twitter Alessio Cortellini @ACortelliniMD, Biagio Ricciuti @BiagioMd, Victor R Vaz@victorrvaz, Joao V Alessi @alessi_joao, Margarita Majem @margamajem, Giuseppe Lamberti @GLambertiMD, Antonio D'Alessio @A_DAlessioMD, Claudia A M Fulgenzi @FulgenziClaudia, Mark M Awad @DrMarkAwad and David J Pinato @ DJPinato

Acknowledgements The authors acknowledge the infrastructure support provided by Imperial Experimental Cancer Medicine Center, Cancer Research UK Imperial Center, and the Imperial College Healthcare NHS Trust Tissue Bank.

Contributors All authors contributed to the publication according to the ICMJE guidelines for the authorship (study conception and design, acquisition of data, analysis and interpretation of data, drafting of manuscript, and critical revision). All authors read and approved the submitted version of the manuscript (and any substantially modified version that involves the author's contribution to the 
study). Each author agreed both to be personally accountable for the author's own contributions and to ensure that questions related to the accuracy or integrity of any part of the work, even ones in which the author was not personally involved, are appropriately investigated, resolved, and the resolution documented in the literature.

Funding This study was supported in part by the NIHR Imperial College BRC Push for Impact scheme 2019. AD'A is supported by grant funding from the European Association for the Study of the Liver (Andrew Burroughs Fellowship). BR is supported by the Society of Immunotherapy of Cancer-AstraZeneca Young Investigator Award. DJP is supported by grant funding from the Wellcome Trust Strategic Fund (PS3416) and from the Associazione Italiana per la Ricerca sul Cancro (AIRC MFAG Grant ID 25697) and acknowledges support by the NIHR Imperial Biomedical Research Centre (BRC), the Imperial Experimental Cancer Medicine Centre (ECMC) and the Imperial College Tissue Bank. AC is supported by the NIHR Imperial BRC.

Disclaimer The views expressed are those of the authors and do not necessarily reflect those of the National Institute for Health Research or the Department of Health and Social Care.

Competing interests $\mathrm{AC}$ received speaker fees and grant consultancies from AstraZeneca, MSD, BMS, Roche, Novartis, and EISAI. GLB received grant consultancies from AstraZeneca and Astellas Pharma. LM reports receiving research grant/funding (self) from Bristol-Myers Squibb, Boehringer Ingelheim, Amgen, Stilla, and Inivata; serving in advisory/consultancy for Roche and Takeda; receiving honoraria (self) from Bristol-Myers Squibb, Takeda, and Roche; receiving travel/accommodation/expenses from Roche, Bristol-Myers Squibb, Takeda, and AstraZeneca; and having non-remunerated activities from AstraZeneca. AA received consulting fees from BMS, AstraZeneca, Boehringer-Ingelheim, Roche, MSD, Pfizer, Eli Lilly, and Astellas; speakers fees from Eli Lilly and AstraZeneca. MT received speaker and consultant fees from AstraZeneca, Pfizer, Eli-Lilly, BMS, Novartis, Roche, MSD, Boehringer Ingelheim, Otsuka, Takeda, Pierre Fabre, Amgen, and Merck. MT received institutional research grants from AstraZeneca, and Boehringer Ingelheim. CC received honorarium/educational grants from Lilly, Pfizer, and Boehringer-Inghelheim. DJP received lecture fees from ViiV Healthcare and Bayer Healthcare and travel expenses from BMS and Bayer Healthcare; consulting fees for Mina Therapeutics, EISAl, Roche, AstraZeneca, DaVolterra, and BMS; and received research funding (to institution) from MSD and BMS. All other authors declare no competing interests.

\section{Patient consent for publication Not applicable.}

Ethics approval This study involves human subjects and was approved by Imperial College Tissue Bank (reference number 17/WA/0161/R18009). Subjects gave informed consent to participate in the study before taking part.

Provenance and peer review Not commissioned; externally peer reviewed.

Data availability statement Data are available upon reasonable request. Availability of data and materials: the datasets used during the present study are available from the corresponding author upon reasonable request.

Supplemental material This content has been supplied by the author(s). It has not been vetted by BMJ Publishing Group Limited (BMJ) and may not have been peer-reviewed. Any opinions or recommendations discussed are solely those of the author(s) and are not endorsed by BMJ. BMJ disclaims all liability and responsibility arising from any reliance placed on the content. Where the content includes any translated material, BMJ does not warrant the accuracy and reliability of the translations (including but not limited to local regulations, clinical guidelines, terminology, drug names and drug dosages), and is not responsible for any error and/or omissions arising from translation and adaptation or otherwise.

Open access This is an open access article distributed in accordance with the Creative Commons Attribution Non Commercial (CC BY-NC 4.0) license, which permits others to distribute, remix, adapt, build upon this work non-commercially, and license their derivative works on different terms, provided the original work is properly cited, appropriate credit is given, any changes made indicated, and the use is non-commercial. See http://creativecommons.org/licenses/by-nc/4.0/.

\section{ORCID iDs}

Alessio Cortellini http://orcid.org/0000-0002-1209-5735

Biagio Ricciuti http://orcid.org/0000-0002-0651-2678
Joao V Alessi http://orcid.org/0000-0002-8072-5946

Margarita Majem http://orcid.org/0000-0002-9919-7485

Giuseppe Lamberti http://orcid.org/0000-0003-3069-7630

Laura Mezquita http://orcid.org/0000-0003-0936-7338

Mark M Awad http://orcid.org/0000-0003-0928-5244

David J Pinato http://orcid.org/0000-0002-3529-0103

\section{REFERENCES}

1 Cortellini A, Bersanelli M, Buti S, et al. A multicenter study of body mass index in cancer patients treated with anti-PD-1/PD-L1 immune checkpoint inhibitors: when overweight becomes favorable. $J$ Immunother Cancer 2019;7:57.

2 Cortellini A, Bersanelli M, Santini D, et al. Another side of the association between body mass index (BMI) and clinical outcomes of cancer patients receiving programmed cell death protein-1 (PD-1)/ Programmed cell death-ligand 1 (PD-L1) checkpoint inhibitors: A multicentre analysis of immune-related adverse events. Eur $J$ Cancer 2020;128:17-26.

3 Wang Z, Aguilar EG, Luna Jl, et al. Paradoxical effects of obesity on $T$ cell function during tumor progression and PD-1 checkpoint blockade. Nat Med 2019;25:141-51.

4 Ichihara E, Harada D, Inoue K, et al. The impact of body mass index on the efficacy of anti-PD-1/PD-L1 antibodies in patients with nonsmall cell lung cancer. Lung Cancer 2020;139:140-5.

5 Kichenadasse G, Miners JO, Mangoni AA, et al. Association between body mass index and overall survival with immune checkpoint inhibitor therapy for advanced non-small cell lung cancer. JAMA Oncol 2020;6:512-8

6 Cortellini A, Ricciuti B, Tiseo M, et al. Baseline BMI and BMI variation during first line pembrolizumab in NSCLC patients with a PD-L1 expression $\geq 50 \%$ : a multicenter study with external validation. $J$ Immunother Cancer 2020;8:e001403.

7 Gandhi L, Rodríguez-Abreu D, Gadgeel S, et al. Pembrolizumab plus chemotherapy in metastatic non-small-cell lung cancer. $N$ Engl J Med 2018;378:2078-92.

8 Paz-Ares L, Luft A, Vicente D, et al. Pembrolizumab plus chemotherapy for squamous non-small-cell lung cancer. $N$ Engl J Med 2018;379:2040-51.

9 Cortellini A, Ricciuti B, Facchinetti F, et al. Antibiotic-exposed patients with non-small-cell lung cancer preserve efficacy outcomes following first-line chemo-immunotherapy. Ann Oncol 2021;32:1391-9.

10 Cortellini A, Di Maio M, Nigro O, et al. Differential influence of antibiotic therapy and other medications on oncological outcomes of patients with non-small cell lung cancer treated with first-line pembrolizumab versus cytotoxic chemotherapy. J Immunother Cancer 2021;9:e002421.

11 Lee KWC, Lord SJ, Kasherman L, et al. The impact of smoking on the effectiveness of immune checkpoint inhibitors - a systematic review and meta-analysis. Acta Oncol 2020;59:96-100.

12 Graff-Iversen S, Hewitt S, Forsén L, et al. Associations of tobacco smoking with body mass distribution; a population-based study of 65,875 men and women in midlife. BMC Public Health 2019;19:1439.

13 Kaufman A, Augustson EM, Patrick H. Unraveling the relationship between smoking and weight: the role of sedentary behavior. $J$ Obes 2012;2012:1-11.

14 El-Osta HE, Mott FE, Burt BM, et al. Predictors of benefits from frontline chemoimmunotherapy in stage IV non-small-cell lung cancer: a meta-analysis. Oncoimmunology 2019;8:e1665974.

15 Dahlberg SE, Schiller JH, Bonomi PB, et al. Body mass index and its association with clinical outcomes for advanced non-small-cell lung cancer patients enrolled on Eastern Cooperative Oncology group clinical trials. J Thorac Oncol 2013;8:1121-7.

16 Argilés JM, Busquets S, Stemmler B, et al. Cancer cachexia: understanding the molecular basis. Nat Rev Cancer 2014;14:754-62.

17 Scott HR, McMillan DC, Forrest LM, et al. The systemic inflammatory response, weight loss, performance status and survival in patients with inoperable non-small cell lung cancer. $\mathrm{Br} J$ Cancer 2002;87:264-7.

18 Dall'Olio FG, Marabelle A, Caramella C, et al. Tumour burden and efficacy of immune-checkpoint inhibitors. Nat Rev Clin Oncol 2021. doi:10.1038/s41571-021-00564-3. [Epub ahead of print: 12 Oct 2021]. 